\title{
Antibacterial Effect of Antibiotic-Saturated Fibrin Sealant; In Vitro Study
}

\author{
Rui Ken Tan ${ }^{1}$, Hyun-Su Lee ${ }^{1}$, Huiling Ma ${ }^{1}$, Hyup-Woo Lee ${ }^{1,2}$, Seung-Kyu Han ${ }^{1}$ \\ ${ }^{1}$ Department of Plastic Surgery, Korea University College of Medicine, Seoul; ' 2Department of Laboratory Medicine, College of Medicine, KangWon National \\ University, Chuncheon, Korea
}

\begin{abstract}
Background: Many chronic wounds have inadequate blood supply around the wounds and systemic antibiotics are less efficient. Topical antiseptic can cause cytotoxicity and irritation or allergic dermatitis. Therefore, topical antibiotics that can sustain their effects in an extended period are often needed to replace topical antiseptics. The present study evaluates the effect of antibiotic-saturated fibrin sealant against Methicillin-Resistant Staphylococcus Aureus (MRSA) by adjusting the concentration of the antibiotic as well as the duration exposure.

Methods: Teicoplanin antibiotic was impregnated in the fibrin disc at concentrations of $50 \mu \mathrm{g} / \mathrm{mL}, 100 \mu \mathrm{g} / \mathrm{mL}, 200 \mu \mathrm{g} /$ $\mathrm{mL}, 400 \mu \mathrm{g} / \mathrm{mL}$ and control $(0 \mu \mathrm{g} / \mathrm{mL})$. The samples were incubated at $37^{\circ} \mathrm{C}$ in $\mathrm{CO}_{2}$ incubator for $7,14,21$, and 28 days. After the incubation period, the antibiotic-saturated fibrin discs were transferred to Mueller-Hinton agar surface scrubs with MRSA. The antimicrobial susceptibility testing was carried out according to Clinical and Laboratory Standards Institution document and using filter papers.

Results: The antibiotic-saturated fibrin disc showed a greatest antibacterial activity during the first week. However, the $50 \mu \mathrm{g} / \mathrm{mL}$ groups fell below suitability within 14 days. Other groups maintained their effect for 28 days, and these 3 groups showed suitable effects.

Conclusion: Antibiotic-loaded fibrin sealants may act as vehicles to deliver antibiotics in a local wound infection. Optimal concentration of antibiotics is $100 \mu \mathrm{g} / \mathrm{mL}$.
\end{abstract}

Keywords: Fibrin sealant, Methicillin-Resistant Staphylococcus Aureus (MRSA), Topical antibiotic

\section{Introduction}

Managing wound infection is an essential component for success in chronic wound healing. Preventing the wound from infection and keeping the wound free from bacteria is a difficult task to archive. In chronic wound infection or deep wound infection, the usual treatment is surgical debridement accompanied by systemic antibiotics for delivering antibiotics to the infection site [1-4].

However, many chronic wounds have inadequate blood supply around the wounds due to underlying disease, tissue necrosis, radiation, and etc. For examples most diabetic patients commonly have poor blood supply on the lower extremity. This raises a question on systemic antibiotic those most likely are less efficient because they cannot penetrate into the wound area due to compromise of blood supply. Therefore, topical antiseptic or antiseptic wound dressing may be sometime more useful because the systemic antibiotics are not very practical. Furthermore, over usage or long-term treatment with topical antiseptics can cause cytotoxicity to host cells especially on fibroblasts, keratinocytes, and endothelial cells which are significantly taking part in wound healing process, causing wound healing process to slow down. In addition, long-term dressing with antiseptics frequently causes irri-

\section{Original Article}

Received: January 25, 2017

Accepted: February 2, 2018

\section{Corresponding author: Seung-Kyu Han, M.D., Ph.D.}

Department of Plastic Surgery, Korea University Guro Hospital, Korea University College of Medicine, 148 Gurodong-ro, Guro-gu, Seoul 08308, Korea

Tel: +82-2-2626-3333

Fax: +82-2-868-6698

E-mail: pshan@kumc.or.kr

This is an Open Access article distributed under the terms of the Creative Commons Attribution Non-Commercial License (http://creativecommons.org/licenses/by-nc/4.0/) which permits unrestricted non-commercial use, distribution, and reproduction in any medium, provided the original work is properly cited.

(c) 2018 Korean Wound Management Society 
tation or allergic dermatitis. Therefore, topical antibiotics that can sustain their effect in an extended period are often needed to replace topical antiseptics, so that wound healing process does not disrupt.

Fibrin sealant is a well-known hemostatic agent that has been known over the past decade. Fibrin sealants consist of fibrinogen and thrombin components, both of which are isolated from human plasma [3]. It has remarkable properties, that turn it into a net-like structure of a fibrin sealant clot with both fibrinogen and thrombin [5], improving wound healing. It is biodegradable and also promotes wound healing without the need to remove it. Since the fibrin sealant is a gel-like liquid, it can be applied to any small space or gap, making it a close to ideal material as a local antibiotic drug delivery vehicle.

Although previously published reports have presented clinical trials and effect of a sustained release of the antimicrobial agents [6,7], the optimal drug concentration and duration for sustained effect against bacteria are still unknown. The present study evaluates the effect of antibiotic-saturated fibrin sealant against Methicillin-Resistant Staphylococcus Aureus (MRSA, ATCC33591; Microbiology Laboratory, Authors' Institution, Seoul, Korea) by adjusting the concentration of the antibiotic as well as the duration exposure.

\section{Methods}

\section{Medium and reagents}

Fibrin sealants (Tisseel ${ }^{\circledR}$; Baxter AG, Wien, Austria), Dulbecco's Modified Eagle and Medium/Ham's F-12 nutrient (DMEM/F-12; Gibco; Grand Island, NY, USA), Mueller-Hinton agar (MHA-100, Korea Medical Holdings, Seoul, Korea) and blood agar (Infusion Agar, Asan Pharmceutical, Seoul, Korea) were purchased separately from their pharmaceutical companies. The antimicrobial drug utilized in this study was Teicoplanin (Ildong Pharm. Co., Seoul, Korea).

\section{Microorganisms}

The MRSA bacteria was placed on blood agar and grown for 24 hours in an incubator with $5 \% \mathrm{CO}_{2}$ incubator at $37^{\circ} \mathrm{C}$. A single colony was harvested into a glass tube with $3 \mathrm{~mL}$ of distilled water and was measured using a Vitek colorimeter to be at a concentration of approximately $1 \times 10^{5}$ colony-forming units $/ \mathrm{mL}$. A thin layer of MRSA was then gently scrubbed and allowed to achieve full coverage of the inoculated on the MHA surface, which was then allowed to dry.

\section{Antibiotic-saturated fibrin disc test}

The teicoplanin antibiotic was impregnated in the fibrin disc at concentrations of $50 \mu \mathrm{g} / \mathrm{mL}, 100 \mu \mathrm{g} / \mathrm{mL}, 200 \mu \mathrm{g} / \mathrm{mL}, 400$ $\mu \mathrm{g} / \mathrm{mL}$, and control $(0 \mu \mathrm{g} / \mathrm{mL})$ in 96 cell culture cluster wells to shape it into a disc-like shape ( $3 \mathrm{~mm} \times 5 \mathrm{~mm}$ ). The fibrin discs were moved into a 24-cell culture well with DMEM/F-12 to obtains the transuded condition, and the samples were incubated at $37^{\circ} \mathrm{C}$ in $\mathrm{CO}_{2}$ incubator for $7,14,21$, and 28 days. After the incubation period, the antibiotic-saturated fibrin discs were transferred to MHA surface scrubs with MRSA $(n=5)$. The antimicrobial susceptibility testing was carried out according to Clinical and Laboratory Standards Institution (CLSI) document M100-S23 (ISBN 1-56238-865-7 [Print]; ISBN 1-56238-866-5 [Electronic]) which is $14 \mathrm{~mm}$ in diameter for teicoplanin antibacterial effect against MRSA.

\section{Filter paper test}

The antibiotic disc was removed from the cell culture cluster well, leaving behind DMEM/F-12 with antibiotic that had been released from the disk at 7, 14, 21, and 28 days. Immediately after, filter paper was cut into round sample with a $5 \mathrm{~mm}$ diameter to match the antibiotic disc and was inserted into the cluster well. The filter papers were incubated for 24 hours at $37^{\circ} \mathrm{C}$ in the $\mathrm{CO}_{2}$ incubator, and the filter papers were inoculated with MHA scrubbed with the MRSA strain. After 24 hours, the diameter of the non-bacterial inhibition zone on the filter paper was measured, and the mean was recorded $(n=5)$.

\section{Results}

Antibiotic-saturated fibrin disc test

The antibiotic-saturated fibrin disc showed a greatest antibacterial activity during the first week. All groups except a control group demonstrated sustained effect until the fourth week (Figs. 1, 2, and Table 1).The antibacterial effect sharply decreased after the first week, but continued to exhibit a regular antibacterial effect up to the fourth week. However the $50 \mathrm{\mu g} /$ $\mathrm{mL}$ groups fell below suitability within 14 days. Other groups maintained their effect for 28 days, and these 3 groups showed suitable effect.

\section{Filter papers test}

After 24 hours of incubation in DMEM/F-12 (after the antibiotic-saturated fibrin disc had been removed) at $37^{\circ} \mathrm{C}$ in a $\mathrm{CO}_{2}$ incubator, all different concentrations showed stable, con- 

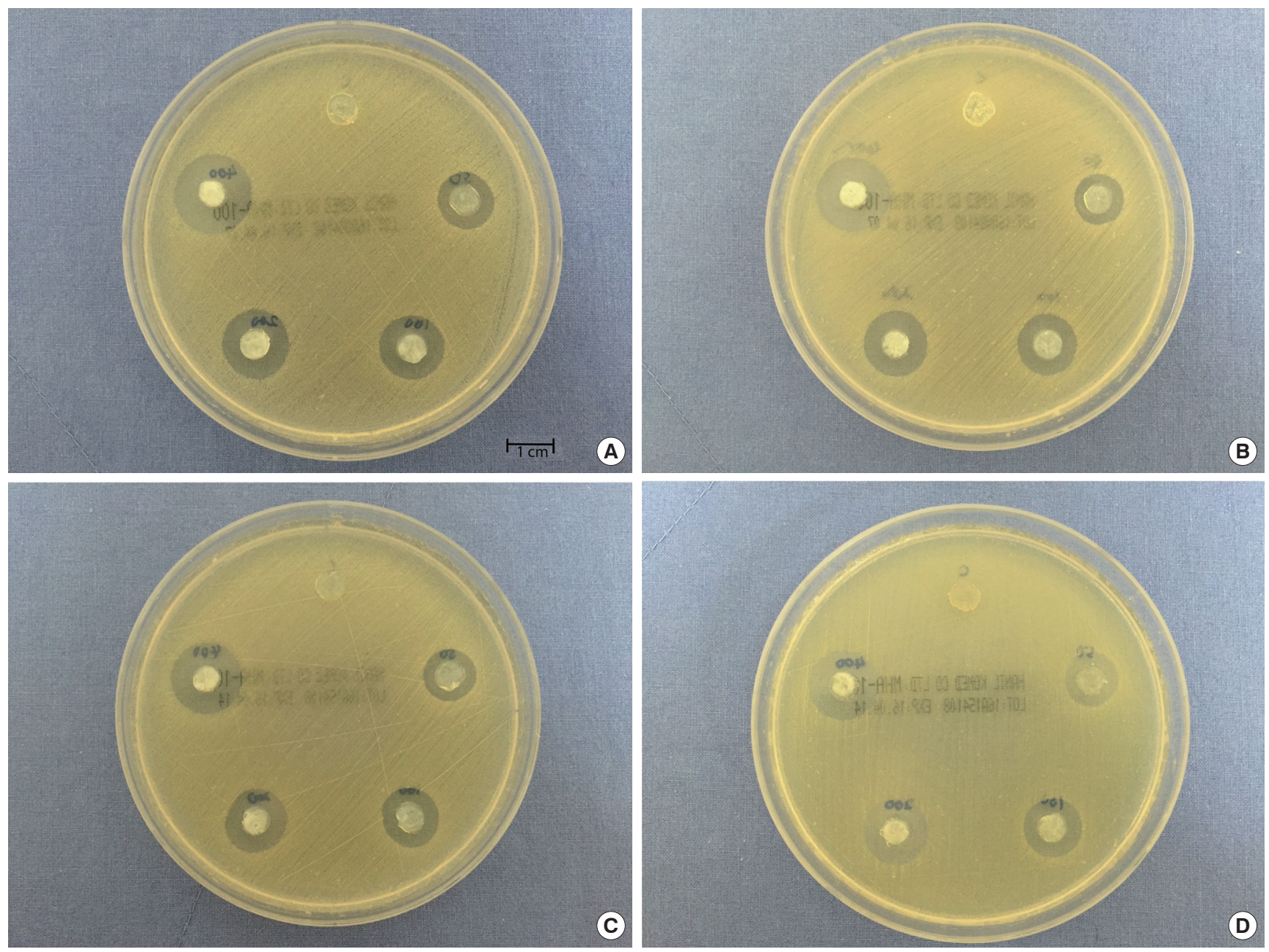

Fig. 1. Zone of inhibition antibiotic-saturated fibrin disc. Zone of inhibition induced by antibiotic-saturated fibrin disc, including control at $0 \mu \mathrm{g} / 100 \mu \mathrm{L}$ (12 o'clock position) and samples $50 \mu \mathrm{g} / 100 \mu \mathrm{L}, 100 \mu \mathrm{g} / 100 \mu \mathrm{L}, 200 \mu \mathrm{g} / 100 \mu \mathrm{L}$, and $400 \mu \mathrm{g} / 100 \mu \mathrm{L}$ (clock-wise direction) against MRSA. (A) First week. (B) Second week. (C) Third week. (D) Fourth week.

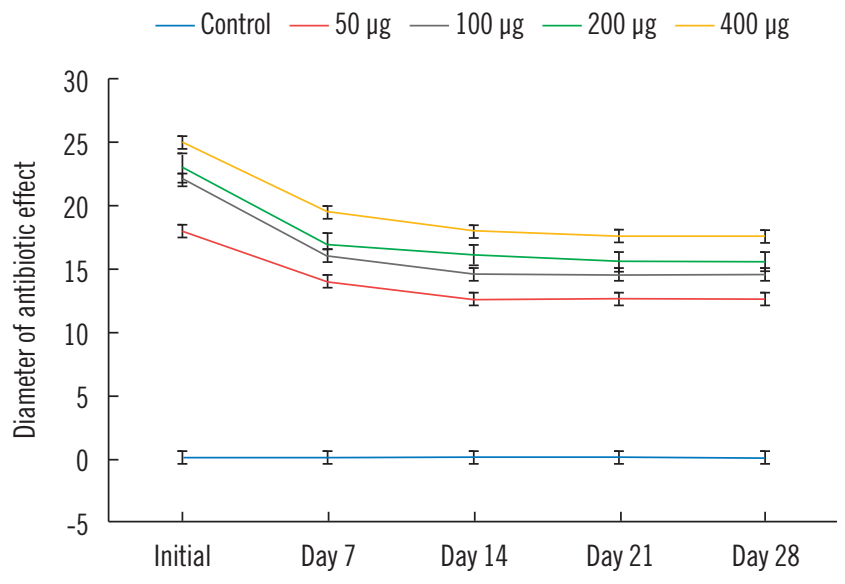

Fig. 2. Inhibition zone of antibiotic-saturated fibrin disc in $\mathrm{mm}$. A graph data showing zone of inhibition antibiotic-saturated fibrin disc in $\mathrm{mm}$. stant release with noticeable antibiotic activity. All four inhibition measurements induced by the filter paper absorbent against MRSA demonstrated a prolonged and sustained release (Figs. 3, 4, and Table 2).

\section{Discussion}

Chronic diabetic foot ulcer has been a major cause of lower limb amputation, and vascular system failure prevents systemic antibiotic form reaching an infection site, causing severe problems that lead to an amputation. Surgical debridement is one of the best options to remove pathologic tissues and may achieve an infection-free wound site. Additionally, it is usually necessary to maintain a high concentration of the appropriate antibiotic at the wound site for a sufficiently long 

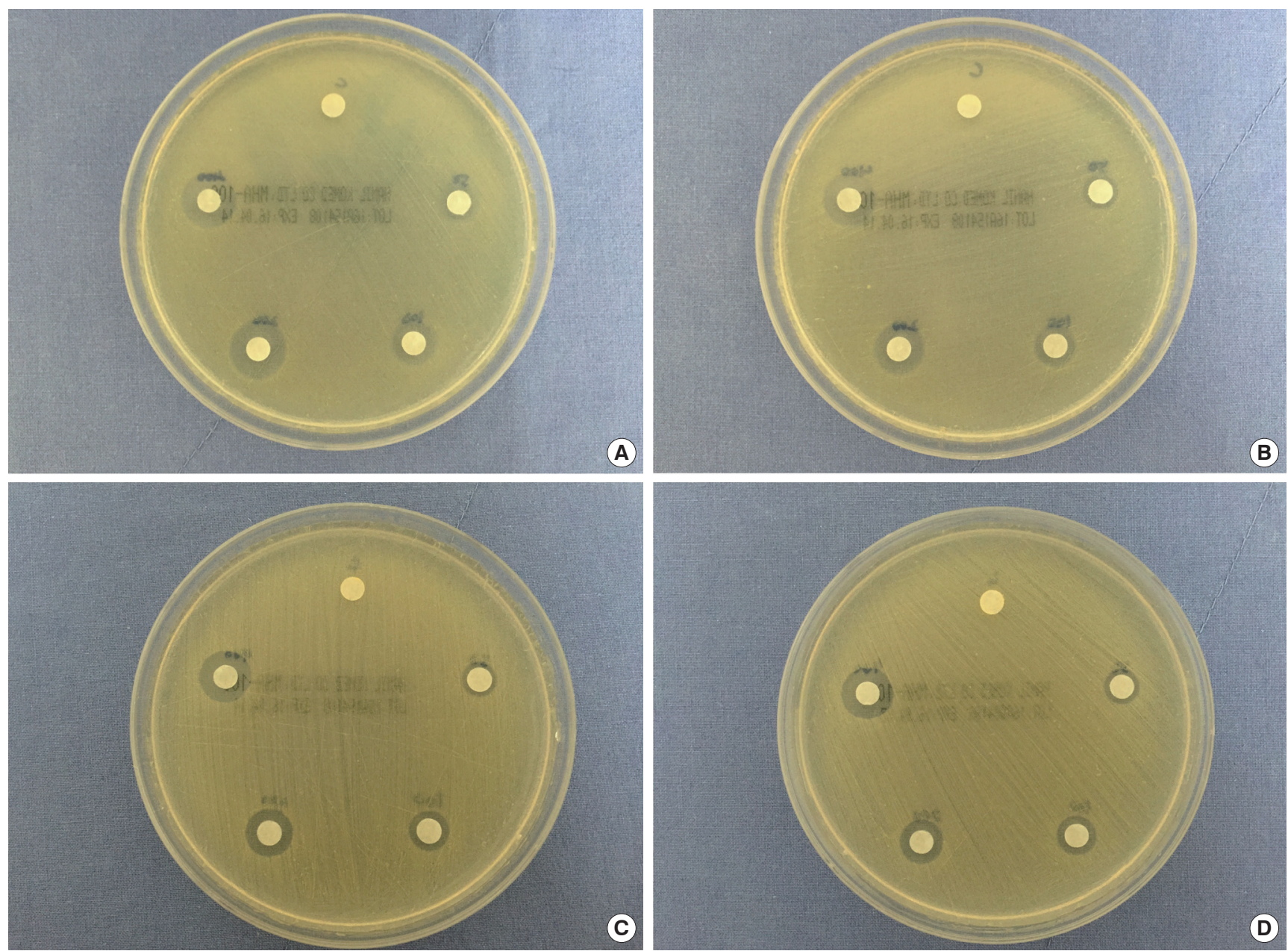

Fig. 3. Zone of inhibition induced by filter paper test. Zone of inhibition induced by filter paper absorbent against MRSA (Refer concentration to Fig. 1; 12 o'clock position low to high clock-wise direction). (A) First week. (B) Second week. (C) Third week. (D) Fourth week.

Table 1. Zone of inhibition induced by antibiotic-saturated fibrin disc against MRSA

\begin{tabular}{lccccc}
\hline \multirow{2}{*}{ Antibiotic concentration } & \multicolumn{5}{c}{ Diameter of non-bacteria inhibition zone $(\mathrm{mm})$} \\
\cline { 2 - 5 } & Initial Time & 7 days & 14 days & 21 days & 28 days \\
\hline Control $(0 \mathrm{mg} / 100 \mathrm{~mL})$ & 0 & 0 & 0 & 0 & 0 \\
$50 \mu \mathrm{g} / 100 \mathrm{~mL}$ & $18 \pm 0.5$ & $14 \pm 0.5$ & $12.5 \pm 0.5$ & $12.5 \pm 0.5$ & $12.5 \pm 0.5$ \\
$100 \mu \mathrm{g} / 100 \mathrm{~mL}$ & $22 \pm 0.5$ & $16 \pm 0.5$ & $14.5 \pm 0.5$ & $14.5 \pm 0.5$ & $14.5 \pm 0.5$ \\
$200 \mu \mathrm{g} / 100 \mathrm{~mL}$ & $23 \pm 0.5$ & $17 \pm 0.5$ & $16 \pm 0.5$ & $15.5 \pm 0.5$ & $15.5 \pm 0.5$ \\
$400 \mu \mathrm{g} / 100 \mathrm{~mL}$ & $25 \pm 0.5$ & $19.5 \pm 0.5$ & $18 \pm 0.5$ & $17.5 \pm 0.5$ & $17.5 \pm 0.5$ \\
\hline
\end{tabular}

time [2].

Poly (methyl methacrylate) antibiotic-impregnated beads are commonly used as prophylactic for local acute or chronic infection. However, poly (methyl methacrylate) only acts as a delivery vehicle for local drug delivery and has to be removed when the antibiotic effect is exhausted [8]. As an alternative, fibrin sealants can also be used as well as to support tissue regeneration or vehicles for delivery for tissue regeneration in chronic wounds $[5,9,10]$. There are many reports on the encapsulation and release of antibiotics from fibrin sealant $[11,12]$, which show it potential as a biocompatible, biodegradable drug delivery system because sealant clots adhere 


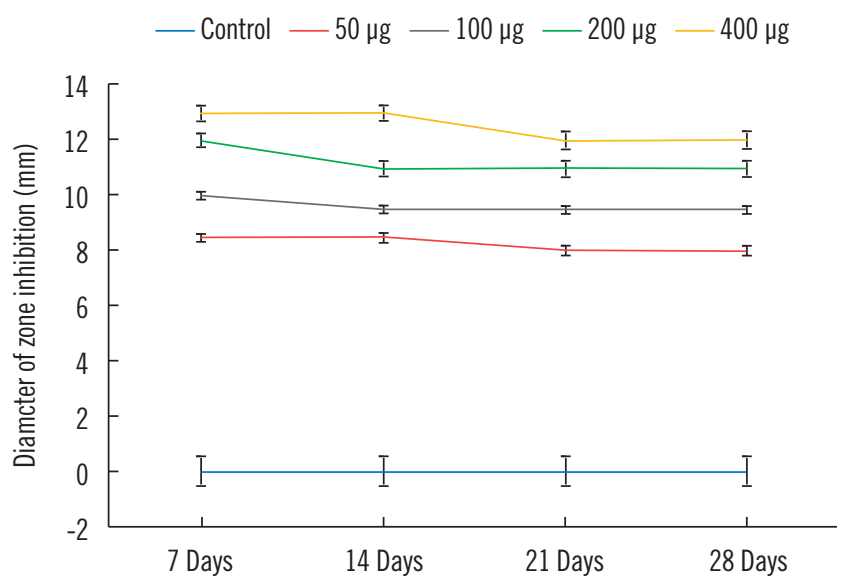

Fig. 4. Filter paper test antibacterial activity. Antibacterial activity of 24 hour's filter paper antibiotic releases test against MRSA.

to the target tissue and allow for controlled release of the drug over an extended period of time [13]. In this study, we used a fibrin sealant saturated with teicoplanin to assess its potential therapeutic activity against MRSA.

In previous studies, some hot topics debate about the moist wound and dry wound healing process. It turned out that moist wound healing has a better outcome than dry wound. In moist condition, wounds tend to heal much faster than in dry wound. Scabs were commonly forming in a dry wound, and it can lock out the oxygen provided from the environment to the wound side especially in inadequate blood supply chronic diabetic foot patients. However, fibrin sealants are a combination of fibrinogen and thrombin that extracted from blood serums. It because of it gel-like properties, fluid can be lock within after both fibrinogen and thrombin are mixed and from fibrin sealants. This is an addition ideal property that fibrin sealants provided to maintain moist to a chronic open wound.

The range of a safe dose for the topical antibiotic administration is important issue to be considered [8]. In this study, we have demonstrated treatment effect with teicoplanin at the given concentrations. MRSA is a resistant strain that is commonly manifested in a chronic infection. In the meantime, we have tried to control the level of the teicoplanin concentration and determine the limitations when controlling the concentration. The fibrin sealant has shown that it can maintain a constant drug release rate. We monitored the drug concentration, but the release rate from the fibrin sealant was unexpected. This in vitro study shows optimal, long-lasting effect for the drugs released for over 28 days. Although the development of drug resistance is a serious concern to sur-
Table 2. Antibacterial activity of filter paper test against MRSA

\begin{tabular}{lcccc}
\hline \multirow{2}{*}{$\begin{array}{c}\text { Antibiotic } \\
\text { concentration }\end{array}$} & \multicolumn{4}{c}{ Diameter of non-bacteria inhibition zone } \\
\cline { 2 - 5 } & 7 days & 14 days & 21 days & 28 days \\
\hline $\begin{array}{c}\text { Control } \\
(0 \mathrm{mg} / 100 \mathrm{~mL})\end{array}$ & 0 & 0 & 0 & 0 \\
$50 \mu \mathrm{g} / \mathrm{mL}$ & $8.5 \pm 0.5$ & $8.5 \pm 0.5$ & $8 \pm 0.5$ & $9 \pm 0.5$ \\
$100 \mu \mathrm{g} / \mathrm{mL}$ & $10 \pm 0.5$ & $9.5 \pm 0.5$ & $9.5 \pm 0.5$ & $9.5 \pm 0.5$ \\
$200 \mu \mathrm{g} / \mathrm{mL}$ & $12 \pm 0.5$ & $11 \pm 0.5$ & $11 \pm 0.5$ & $11 \pm 0.5$ \\
$400 \mu \mathrm{g} / \mathrm{mL}$ & $13 \pm 0.5$ & $13 \pm 0.5$ & $12 \pm 0.5$ & $12 \pm 0.5$ \\
\hline
\end{tabular}

geons, we do not believe that this method of topical application of a controlled-release formulation of would exacerbate this problem [13].

In CLSI document M100-S23 the antibiotic susceptibility test indicated that $14 \mathrm{~mm}$ are the susceptibility value of teicoplanin antibacterial effect for MRSA. From the result shown (Fig. 2), $100 \mu \mathrm{g} / \mathrm{mL}, 200 \mu \mathrm{g} / \mathrm{mL}$ and $400 \mu \mathrm{g} / \mathrm{mL}$ have surpassed the antimicrobial susceptibility test. Therefore, the optimal concentration from this study can be $100 \mu \mathrm{g} / \mathrm{mL}$. We did mention that developments of drug resistance are the concern for all healthcare parties; $100 \mu \mathrm{g} / \mathrm{mL}$ should be used as an optimal concentration for topical antibiotic delivery may prevent manifestation of drug resistant in the near future.

Despite that teicoplanin-saturated fibrin discs displayed potential therapeutic activity against the MRSA stain, lyses and absorption of the fibrin sealant [14] were not tested in this study. The absorption of fibrin sealant is relatively important for the antibacterial effect to be prolonged, and we believe that the faster the fibrin sealant absorbs, the more rapid drug will be depleted.

In the previous study, Selçuk Kara mentioned that specific antibiotic only lasts for 48 hours. In our in vitro study show that teicoplanin had continued for 28 days. Of cause, the concentration that we had tested are different despised that they did not mention about the size or thickness of the fibrin sealant. Our study has shown support that the antimicrobial actually could last for 28 days. However, if this in vitro applies in animal or clinical, there are few conditions cannot be ignored. Every single person has differences in DNA, blood supply, etc. Therefore, the expectation antibacterial effect might be shorter as the absorption or lyses effect kick in.

There are limitations in this study. The microorganisms and antibiotic tested in this in vitro mode are not universal. In a further study, more antibiotics and variations in antimicrobial resistance bacteria need to be assessed. In addition, the ab- 
sorption of fibrin sealant should be tested with the release of antibiotic effect in an animal study. Of course, clinical study should be major consider in the future for more information and data can be a valuable asset in chronic wound healing.

The results of this study show the possibility that antibiotic could be mixed with fibrin sealant to treat wound infections with inadequate of blood supply. Limited concentrations of antibiotics would be mixed with fibrin sealant without over using high-concentration antibiotics. Fibrin sealant may be applied in an infected site, not only for tissue regeneration but also to control an infection [2].

Fibrin sealants provided a prolonged drug delivery, which indicates that antibiotic-saturated fibrin clots act as vehicles to deliver the drug in a local wound infection by controlling the concentration of drug used.

\section{Conflict of interest}

No potential conflict of interest relevant to this article was reported.

\section{Acknowledgment}

This article was presented as a poster at the International 74th Congress of the Korean Society of Plastic and Reconstructive Surgeons on Nov 17-20, 2016.

\section{References}

1. Boyce ST, Holder IA, Supp AP, et al. Delivery and activity of antimicrobial drugs released from human fibrin sealant. J Burn Care Rehabil 1994;15(3):251-5.

2. Park MS, Kim YB. Sustained release of antibiotic from a fibrin-gelatin-antibiotic mixture. Laryngoscope 1997;107(10): 1378-81.

3. Schultz GS, Sibbald RG, Falanga V, et al. Wound bed prepa- ration: a systematic approach to wound management. Wound Repair Regen 2003;11 Suppl 1:S1-28.

4. Marone P, Monzillo V, Segù Ç, et al. Antibiotic-impregnated fibrin glue in ocular surgery: in vitro antibacterial activity. Ophthalmologica 1999;213(1):12-5.

5. Macasev D, Diorio JP, Gugerell A, et al. Cell compatibility of fibrin sealants: in vitro study with cells involved in soft tissue repair. J Biomater Appl 2011;26(2):129-49.

6. Nakaminami H, Suzuki Y, Suzuki R, et al. In vitro antimicrobial activity of fibrin sealants containing antimicrobial agents. Surg Infect (Larchmt) 2014;15(1):29-35.

7. Kara S, Vural A, Unver A, et al. Fibrin sealant as a carrier for sustained delivery of antibiotics. J Clin Exp Invest 2014;5(2).

8. Mader JT, Stevens CM, Stevens JH, et al. Treatment of experimental osteomyelitis with a fibrin sealant antibiotic implant. Clin Orthop Relat Res 2002(403):58-72.

9. Michel D, Harmand MF. Fibrin seal in wound healing: effect of thrombin and $[\mathrm{Ca} 2+]$ on human skin fibroblast growth and collagen production. J Dermatol Sci 1990;1(5):325-33.

10. Fujioka-Kobayashi M, Mottini M, Kobayashi E, et al. An in vitro study of fibrin sealant as a carrier system for recombinant human bone morphogenetic protein (rhBMP)-9 for bone tissue engineering. J Craniomaxillofac Surg 2017;45(1):27-32.

11. Thompson DF, Davis TW. The addition of antibiotics to fibrin glue. South Med J 1997;90(7):681-4.

12. Redl H, Schlag G, Stanek G, et al. In vitro properties of mixtures of fibrin seal and antibiotics. Biomaterials 1983;4(1): 29-32.

13. Tredwell S, Jackson JK, Hamilton D, et al. Use of fibrin sealants for the localized, controlled release of cefazolin. Can J Surg 2006;49(5):347-52.

14. Pflüger H. Lysis and Absorption of Fibrin Sealant (Tissucol/ Tisseel). In: Schlag G, Redl H, editors. Fibrin Sealant in Operative Medicine: Volume 6 General Surgery and Abdominal Surgery. Berlin, Heidelberg: Springer Berlin Heidelberg; 1986. p. 39-50. 